\title{
DETERMINACIÓN DE LA VELOCIDAD DE LOS GASES EN EL SUBSUELO MEDIANTE UN MÉTODO BASADO EN LAS VARIACIONES DE LA CONCENTRACIÓN DEL GAS RADÓN
}

\author{
J. R. García-Vindas \\ Universidad de Costa Rica, Escuela de Física, Sección de Física Nuclear Aplicada \\ San Pedro de Montes de Oca, San José, Costa Rica \\ E-mail: jrgarcia@cariari.ucr.ac.cr
}

(Recibido 19/10/2000; Aceptado 14/5/2001)

\begin{abstract}
In this paper a theoretic model is proposed to calculate the gas velocity in the subsoil based on radon concentration variations. The general transport equation for radon in a homogeneous soil with constant porosity is assumed. The diffusion coefficient and the gas velocity being constant. In order to illustrate the model, three geological areas were considered: the Irazú and Arenal volcanoes, situated in the volcanic range in Costa Rica, and the Agua Caliente fault located in San José, Costa Rica.
\end{abstract}

\begin{abstract}
RESUMEN: En este trabajo se propone un modelo teórico para la determinación de la velocidad de los gases en el subsuelo, basado en las variaciones de la concentración de gas radón. Se parte de la ecuación de transporte para el gas radón y se asume un medio homogéneo con porosidad uniforme e independiente del tiempo. El coeficiente de difusión del radón y la velocidad de transporte permanecen constantes. Para ilustrar el modelo, los cálculos se aplican a tres zonas geológicas diferentes: el Volcán Irazú, el Volcán Arenal, situados en la Cordillera Volcánica Central de Costa Rica y la falla de Agua Caliente, situada en Desamparados, San José, Costa Rica.
\end{abstract}

\section{INTRODUCCIÓN}

Las emisiones gaseosas que escapan de la superficie terrestre, son el resultado de la difusión o del transporte convectivo, debido a diferencias de presión. Estas emisiones se componen principalmente por vapor de agua, dióxido de carbono y otras especies químicas volátiles, presentes a nivel de trazas, tales como helio y radón. Muchas de las investigaciones realizadas en relación con esos gases consisten, por un lado, en el estudio de las variaciones de la concentración de los mismos a varias pro- fundidades con el fin de encontrar una relación entre estos parámetros con eventos de origen sísmico o volcánico (Garcia-Vindas et al., 2000, Notsu et al., 1991, King, 1984) y por otro lado, en la medición de su flujo total a través de la superficie terrestre (Wilkening, 1974, Fleischer, 1980). Sin embargo existe gran dificultad cuando se mide directamente la componente convectiva del flujo total, debido a la baja sensibilidad de los instrumentos de medida, ya que estos no son capaces de medir la velocidad de los gases presentes en el subsuelo, que es del orden de $10^{-4}-10^{-6} \mathrm{~m} \mathrm{~s}^{-1}$. 
Este trabajo propone un método para calcular la velocidad de los gases en el subsuelo, basado en un modelo teórico y utilizando el gas radón como trazador.

Se pretende mostrar como, a través de las variaciones de la concentración del radón se puede determinar teóricamente la velocidad del fluido que lo transporta (generalmente este fluido es un gas). Este trabajo es complemento de un trabajo anterior realizado por García et al. (2000) en el cual se estableció que las anomalías en la concentración de radón se deben a cambios en el flujo convectivo de este, producto de la migración de fluidos presurizados que lo transportan hacia la superficie.

Como aplicaciones de este método se dan tres ejemplos de zonas geológicas diferentes: la loma de Salitral en Desamparados, el Volcán Irazú y el Volcán Arenal, Costa Rica.

\section{CÁLCULO DE LA VELOCIDAD A PARTIR DE LAS VARIACIONES EN LA CONCENTRACIÓN DE RADÓN}

Considérese el suelo como un medio poroso, homogéneo e isotrópico a través del cuál el radón se transporta por difusión y por convección hacia la superficie. Considérese que en un intervalo de tiempo dado, el flujo total (convectivo + difusivo) se encuentra en estado estacionario, de manera que la concentración de radón se rige por la siguiente ecuación (Clements y Wilkening, 1974):

$$
\frac{D}{\varepsilon} \frac{\partial^{2} C}{\partial z^{2}}-\frac{\mathbf{v}}{\varepsilon} \frac{\partial C}{\partial z}-\lambda C+Q=0
$$

En la ecuación (1), C representa la concentración de radón $\left(\mathrm{Bq} \cdot \mathrm{m}^{-3}\right), \mathrm{D}$ es el coeficiente de difusión $\left(\mathrm{m}^{2} \cdot \mathrm{s}^{-1}\right)$, v la velocidad de Darcy $\left(\mathrm{m} \cdot \mathrm{s}^{-1}\right), \varepsilon$ la porosidad del medio y $\lambda$ la constante de desintegración del radón $\left(\mathrm{s}^{-1}\right)$. Las variaciones en la concentración se dan en la coordenada $\mathrm{z}$, siendo esta la coordenada vertical, de manera que se considera que hay simetría en X Y.

Para resolver la ecuación (1) considérense las siguientes condiciones de frontera:
(2) $\mathrm{C}(\mathrm{z}=0)=0,(\mathrm{z}=0$ representa la superficie del suelo)

$\mathrm{C}(\mathrm{z}=\infty) \mathrm{C}_{\infty}$

De (2) se establece que la concentración de radón en la superficie del suelo (interface suelo-atmósfera) es siempre nula debido a que ahí es varios órdenes de magnitud más pequeña que la concentración en el suelo. También se considera que a grandes profundidades esta permanece constante, de acuerdo con el trabajo de Fleisher \& Mogro-Campero (1979).

La solución de la ecuación (1) tomando en consideración las condiciones de frontera impuestas en (2) viene dada como sigue

$$
C(z)=C_{\infty}\left(1-e^{A z}\right) \mathrm{z}<0
$$

Donde A es:

$$
A=\frac{\mathbf{v}}{2 D}+\sqrt{\left(\frac{\mathbf{v}}{2 D}\right)^{2}+\frac{\lambda \varepsilon}{D}}
$$

Considérese que en un cierto intervalo de tiempo la concentración de radón en cada punto $\mathrm{Z}$ satisface la ecuación (3) con una velocidad constante $\mathrm{v}=\mathrm{v}_{1}$ de manera que la concentración medida en un punto tal como $\mathrm{z}=-1 \mathrm{~m}$ ( a esta profundidad se acostumbra medir generalmente la concentración de radón en el suelo) se expresa como:

$$
C_{1}=C_{\infty}\left(1-e^{-A_{1}}\right)
$$

En la ecuación (5) $A_{1}$ es función de $v_{1}$ :

$$
A_{1}=\frac{\mathbf{v}_{1}}{2 D}+\sqrt{\left(\frac{\mathbf{v}_{1}}{2 D}\right)^{2}+\frac{\lambda \varepsilon}{D}}
$$

Si el sistema permanece en este estado durante un largo tiempo de manera que la concentración en $\mathrm{z}=-1 \mathrm{~m}$ presenta únicamente fluctuaciones estadísticas alrededor de un valor medio igual a $\mathrm{C}_{1}=\mathrm{C}(\mathrm{z}=-1 \mathrm{~m})$.

$\mathrm{Si}$ en un instante dado la componente convectiva del flujo total varía debido a una fluctuación 
en la velocidad de transporte, la concentración de radón en cada punto (Fleischer \& Mogro-Campero, 1979), y por consiguiente en $Z=-1 \mathrm{~m}$, cambiaría.

Entonces la concentración en $\mathrm{Z}=-1$ pasa de $\mathrm{C}_{1}$ a un nuevo valor $\mathrm{C}_{2}$ producto del nuevo valor de $v=v_{2}$. De la ecuación (3) se obtiene:

$$
C_{2}=C_{\infty}\left(1-e^{-A_{2}}\right)
$$

Donde $\mathrm{A}_{2}$ se determina a partir de la relación (6) al sustituir $\mathrm{v}_{1}$ por $\mathrm{v}_{2}$.

El sistema permanece como anteriormente en este estado, durante un largo período, con su concentración en $\mathrm{Z}=-1 \mathrm{~m}$ oscilando estadísticamente alrededor del valor $\mathrm{C}_{2}$. Nuestro problema consiste en determinar $\mathrm{v}_{2}$ a partir de $\mathrm{v}_{1}, \mathrm{C}_{1} \mathrm{y} \mathrm{C}_{2}$. Esto se logra combinando las ecuaciones (5) y (7) para obtener:

$$
\mathbf{v}_{2}=D|B|-\frac{\lambda \varepsilon}{|B|}
$$

donde

$$
B=\ln \left[1-\frac{C_{2}}{C_{1}}\left(1-e^{-A_{1}}\right)\right]<0
$$

En la ecuación (9) las cantidades $\mathrm{C}_{1}, \mathrm{C}_{2}$, $\mathrm{D}, \varepsilon$ y $\lambda$ se pueden estimar o medir. Además se parte de que la velocidad $\mathrm{v}_{1}$ es una cantidad conocida.

Si se considera ahora el caso más simple, cuando $\mathrm{v}_{1}=0$ el flujo inicial sería puramente difusivo, entonces $\mathrm{A}_{1}$ vendría dado como:

$$
A_{1}=\sqrt{\frac{\lambda \varepsilon}{D}}
$$

y la velocidad $v_{2}$ se expresa de la siguiente forma:

$$
\mathbf{v}_{2}=D\left|\ln \left[1-\frac{C_{2}}{C_{1}}\left(1-e^{-\sqrt{\frac{\lambda \varepsilon}{D}}}\right)\right]\right|-\frac{\lambda \varepsilon}{\left|\ln \left[1-\frac{C_{2}}{C_{1}}\left(1-e^{-\sqrt{\frac{\lambda \varepsilon}{D}}}\right)\right]\right|}
$$

Es importante notar aquí que de la ecuación (9) se desprende que el argumento del logaritmo será menor que 1 siempre y cuando $\mathrm{C} 2<$ $\mathrm{C} 1$. En el caso de que $\mathrm{C} 2>\mathrm{C} 1$, la razón entre el coeficiente de difusión y la porosidad deben satisfacer la siguiente condición:

$$
\frac{D}{\varepsilon}>\frac{\lambda}{\left[\ln \left(1-\frac{C_{1}}{C_{2}}\right)\right]^{2}}
$$

Conociendo o fijando el valor de la porosidad, la ecuación (12) suministra una condición necesaria que debe cumplir el coeficiente de difusión cuando se realizan los cálculos.

De acuerdo a ley de Darcy, la velocidad de transporte se expresa en función del gradiente de presión como:

$$
v=-\frac{\kappa \partial P}{\mu \partial z}
$$

Donde $\kappa$ es la permeabilidad del medio $\left(\mathrm{m}^{2}\right), \mu$ la viscosidad del fluido $(\mathrm{Pa} \cdot \mathrm{s})$, que en nuestro caso consiste en una mezcla gaseosa y por ultimo, P representa la presión del fluido $(\mathrm{Pa})$. Combinando las ecuaciones (11) y (13) se obtiene:

$$
\text { (14) } \frac{\partial P}{\partial z}=\frac{\mu}{\kappa}\left\{\frac{\lambda \varepsilon}{\mid \ln \left[1-\frac{C_{2}}{C_{1}}\left(1-e^{\left.-\sqrt{\frac{\lambda \varepsilon}{D}}\right)}\right) \mid\right.}-\right.
$$

La expresión (14) nos permite calcular el gradiente de presión al cual está sometido el fluido, conociendo de antemano la permeabilidad y la viscosidad. 


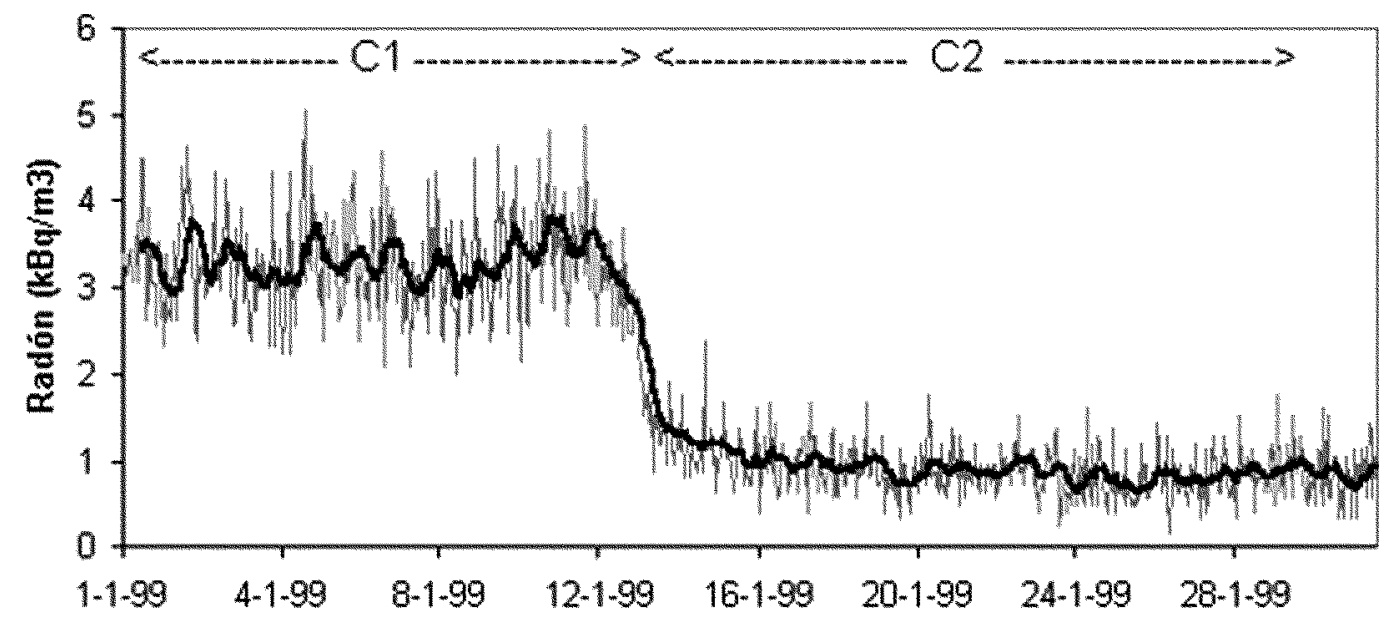

Fig. 1: Concentración de radón en la loma de Salitral, Desamparados (Costa Rica). La curva de trazo fuerte representa la curva de tendencia de los datos, calculada mediante el método de la media móvil para un período de 12 horas.

\section{APLICACIÓN DEL MÉTODO UTILIZANDO DATOS DE CAMPO}

Con el fin de ilustrar la aplicación del modelo expuesto anteriormente se escogieron tres casos en zonas diferentes, en las cuales se midió la concentración de radón en el suelo, a un metro de profundidad y en intervalos de una hora. Estas medidas se realizaron con una sonda electrónica llamada Clipperton, cuya descripción y funcionamiento han sido detallados por Monnin \& Seidel, (1998).

Como primer caso se presenta los datos obtenidos en la loma de Salitral en Desamparados (San José, Costa Rica). Esta loma se encuentra rodeada por las fallas de Agua Caliente y Río Azul, las cuales presentan una sismicidad activa con sismos, por lo general, de magnitud menor que 4 en la escala Richter (Fernández, 1999). La falla de Agua Caliente es la mayor y es de origen neotectónico produciendo esfuerzos extensivos y compresivos en el área. En este caso $\mathrm{C}_{1}=4 \mathrm{y} \mathrm{C}_{2}$ $=1 \mathrm{kBq} / \mathrm{m}^{3}$ (Fig. 1). Estos valores fueron obtenidos promediando los datos en cada uno de los intervalos mostrados en la figura 1. La porosidad del suelo se calculó en el laboratorio y su valor fue de 0.45 y el coeficiente de difusión se estimó en $10^{-6} \mathrm{~m}^{2} / \mathrm{s}^{-1}$. Asumiendo que la concentración $\mathrm{C}_{1}$ obedece a un estado puramente difusivo $\left(\mathrm{v}_{1}=\right.$
0) y en virtud de la ecuación (11) se obtiene que $\mathrm{v}_{2}$ tiene el valor de $-5,53 \times 10^{-6} \mathrm{~m} \cdot \mathrm{s}^{-1}$. El signo negativo indica que la dirección del flujo es hacia el interior de la superficie del suelo.

Como segundo caso se muestra una serie de datos recolectados en el volcán Irazú, bajo las mismas condiciones que en el ejemplo precedente. El volcán Irazú se encuentra localizado en la Cordillera Volcánica Central de Costa Rica y tiene una elevación de $3432 \mathrm{~m}$. Su último período eruptivo comenzó en 1962, presentando desde entonces varias crisis sísmicas y algunas erupciones. En este caso, $\mathrm{C}_{1}=22 \mathrm{y} \mathrm{C}_{2}=34 \mathrm{kBq} \mathrm{m}^{-3}$ (Fig. 2). Se asumen los mismos valores para la porosidad y el coeficiente de difusión que en el caso anterior, el cual está en concordancia con la ecuación (12). Partiendo de la suposición de que el estado inicial es un estado puramente difusivo $\left(\mathrm{v}_{1}=0\right)$ y el valor de $\mathrm{v}_{2}$ obtenido fue de $2.95 \times 10^{-6} \mathrm{~m} \cdot \mathrm{s}^{-1}$.

Como última aplicación, se presenta una serie de datos tomados en el Volcán Arenal y siempre bajo las mismas condiciones anteriores. El volcán Arenal es un estrato-volcán ubicado $90 \mathrm{~km}$ al noroeste de San José, capital de Costa Rica. Se localiza en $10^{\circ} 27^{\prime} 48^{\prime \prime} \mathrm{N}$ y $84^{\circ} 42^{\prime} 12^{\prime \prime} \mathrm{W}$, a $1633 \mathrm{~m}$ sobre el nivel del mar. $\mathrm{Ha}$ presentado actividad permanente desde 1968, con la emisión continua de gases, coladas 


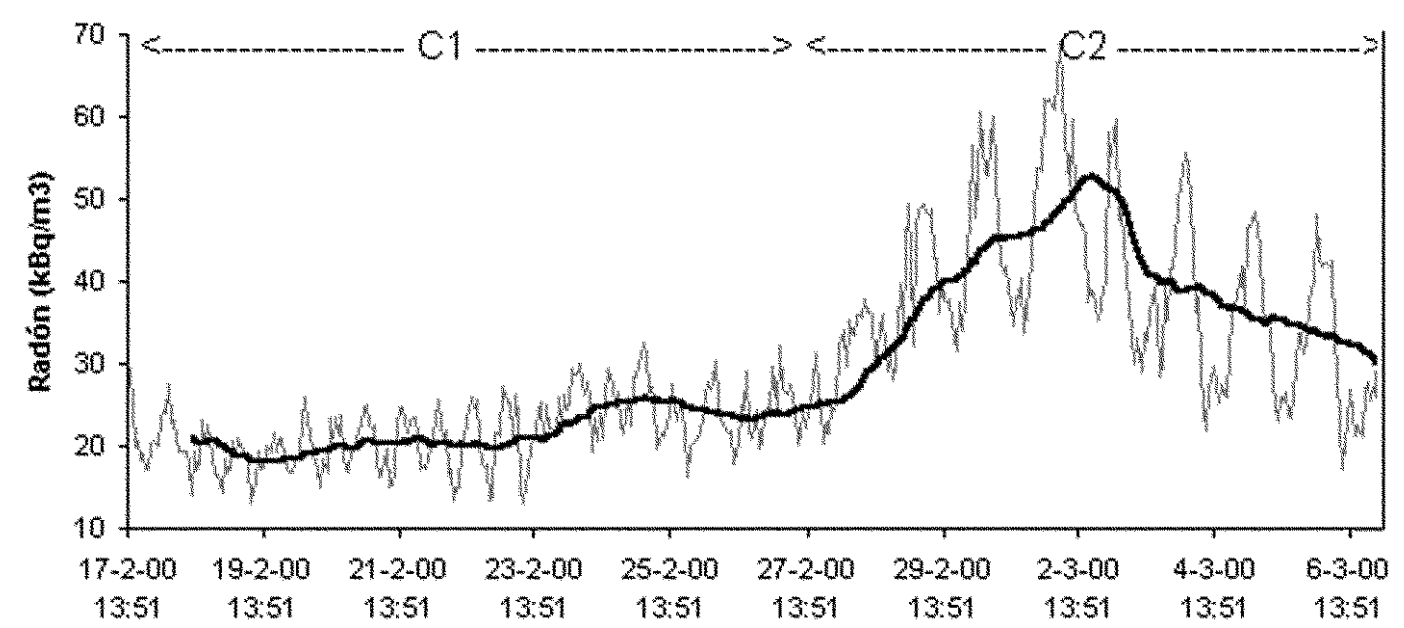

Fig. 2: Concentración de radón en el volcán Irazú (Costa Rica). La curva de trazo fuerte representa la curva de tendencia de los datos, calculada mediante el método de la media móvil para un período de 12 horas.

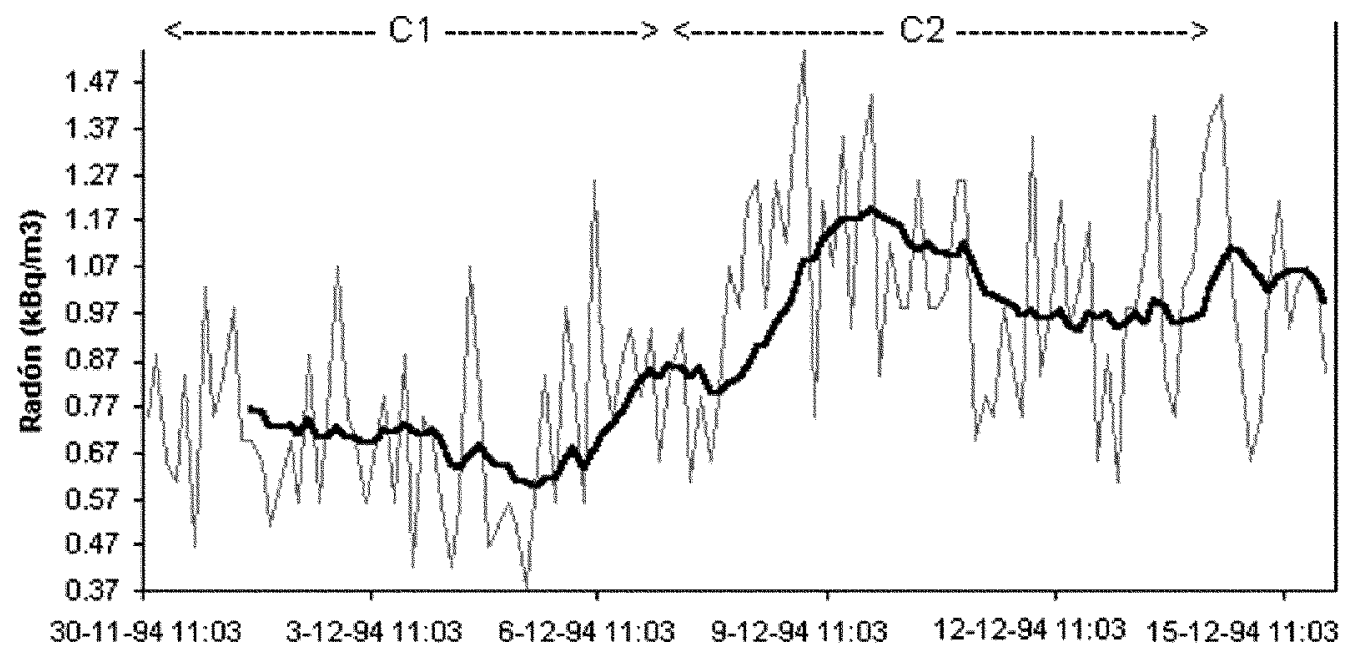

Fig. 3: Concentración de radón en el volcán Arenal (Costa Rica ). La curva de trazo fuerte representa la curva de tendencia de los datos, calculada mediante el método de la media móvil para un período de 12 horas.

de lava, esporádicas erupciones estrombolianas y ocasionales flujos piroclásticos.

Para este caso $\mathrm{C}_{1}=0,73$ y $\mathrm{C}_{2}=1,04$ $\mathrm{kBq} / \mathrm{m}^{3}$. Los valores de porosidad y el coeficiente de difusión tienen los mismos valores que en los dos casos precedentes. La velocidad $\mathrm{v}_{2}$ es de $3,14 \times 10^{-4} \mathrm{~m} / \mathrm{s}^{-1}$ y se calculó asumiendo que el valor $\mathrm{C}_{1}$ pertenece al estado estacionario.

\section{CONCLUSIONES}

El método expuesto en este trabajo constituye una herramienta práctica para solventar el problema de la medición de las velocidades de transporte de los gases del suelo. Como se mencionó en la introducción, este tipo de mediciones es casi imposible de realizar debido a que 
las velocidades de los gases son muy pequeñas $\left(10^{-4}-10^{-6} \mathrm{~m} \cdot \mathrm{s}^{-1}\right)$ para que puedan ser medidas con precisión.

Si se dispone de los valores de la porosidad del suelo y del coeficiente de difusión, es factible obtener la velocidad del gas si se considera que estos parámetros no cambian con el tiempo y que además, el sistema se encuentra en estado estacionario. Si se asume un estado inicial puramente difusivo, entonces la velocidad del gas viene dada por la expresión (11). Por otro lado, si se conocen los valores de permeabilidad y viscosidad del medio, es posible calcular, por medio de la ecuación (14), el gradiente de presión que origina el movimiento del gas.

La ecuación (12) constituye una restricción importante al valor del coeficiente de difusión y es un indicativo para valorar si la condición de estado difusivo puro se puede aplicar a un caso dado. Además, mediante un análisis más detallado, basado en esta restricción, se puede obtener también la velocidad $\mathrm{v}_{1}$, o al menos acotarla superiormente.

De los tres casos estudiados, se desprende que los resultados obtenidos se encuentran dentro del intervalo de velocidades mencionado.

En aquellos lugares donde el radón es escaso se podría modificar el procedimiento de las medidas de este, introduciendo una fuente natural de radón (tal como la pechblenda) en el punto de medición, con el fin de aumentar la concentración a nivel del detector y obtener medidas más precisas. El gas que viaja hacia fuera o hacia adentro de la superficie, transportaría al radón con él a la misma velocidad, aumentando o disminuyendo la concentración local en el detector. Se debe mencionar aquí que se deben usar detectores capaces de medir el radón en forma continua, tales como las sondas Clipperton, y con una gran velocidad de respuesta ante los cambios súbitos en la concentración, con el objeto de calcular con mayor precisión las velocidades de transporte. En nuestro caso, ha sido demostrado que las sondas Clipperton reúnen las condiciones antes mencionadas (GarcíaVindas, 1999).

\section{AGRADECIMIENTOS}

El autor agradece a la Universidad de Costa Rica, en especial a la Vicerrectoría de Investigación, por el apoyo brindado, a través del proyecto "Radón y Sismicidad en Costa Rica," así como a la Universidad de Montpellier II y al Organismo Internacional de Energía Atómica.

\section{REFERENCIAS}

CLEMENTS, W. \& WILKENING, M., 1974: Atmospheric pressure effects on Rn 222 transport across the Earthair interface. - J. Geophys. Res. 29 (33): 5025-5029.

FLEISCHER, R.L., 1980: Radon flux from the earth: methods of measurement by the nuclear track technique. - J. Geophys. Res. 8 (C12): 7553-7556.

FLEISCHER, R.L. \& MOGRO-CAMPERO, A., 1979: Radon enhancements in the earth: evidence for intermittent upflows? - Geophys. Res. Lett. 6 (5): 361-364.

GARCÍA-VINDAS, R., 1999: Transport du radon en milieu poreux (expérimentation et modélisation): implication pour la réalisation et l'interprétation de mesures in situ. - 270 págs. Universidad de Montpellier II [Tésis PhD].

GARCÍA-VINDAS, R., LORÍA, L. G. \& JIMÉNEZ, R., 2000: Ejemplos de anomalías del radón 222, como precursoras de eventos sísmicos en Costa Rica. Rev. Geol. América Central, 23: 87-96.

GARCIA, R., NATALE, G., MONNIN, M. \& SEIDEL, J-L., 2000: Shock waves radon surfaces signals associated with the upsurge of T-P solitons in volcanic systems. - J. Volcanol. Geotherm. Res. 96: 15-24.

KING, C.Y., 1984: Impulsive radon emanation on a creeping segment of the San Andreas fault, California. - Pageoph. 122: 340-351.

MONNIN, M., \& SEIDEL, J., 1998: An automatic radon probe for Earth Science studies. - J. Appl. Geophys. 39: 209-220.

NOTSU, K., WAKITA, H., IGARASHI, G. \& SATO, T., 1991: Hydrological and geochemical changes related to the 1989 seismic and volcanic activities of the Izu Peninsula. - J. Phys. Earth, 39: 245-254.

WILKENING, M.H., 1974: Radon-222 from the island of Hawaii: Deep soils are more important than lava fields or volcanoes. - Science, 183: 413-415. 